\title{
Monitoramento de Tempestades através de Radar Meteorológico no SipamHidro
}

\author{
Marissa B. de Carvalho ${ }^{1}$, Alen C. Vieira ${ }^{1}$, Michell C. O. da Cruz ${ }^{1}$, Anderson F. de \\ Nazaré ${ }^{\text {, Flávio A. dos Santos }}{ }^{1}$, Daniele N. Dias ${ }^{1}$ \\ ${ }^{1}$ Centro Gestor e Operacional do Sistema de Proteção da Amazônia (Censipam) - \\ 66.617-420 - Belém - PA - Brasil \\ $\{$ marissa.carvalho, alen.vieira, michell.cruz, anderson.nazare, flavio.santos, \\ daniele.nogueira\}@sipam.gov.br
}

\begin{abstract}
Resumo. O SipamHidro é um sistema do Censipam que possui um conjunto de tecnologias para auxiliar o monitoramento, análise e previsão de informações espaciais das condições hidrológicas e meteorológicas. Um dos seus módulos é o Radar Meteorológico que fornece informações sobre diferentes parâmetros meteorológicos. Este trabalho apresenta-o, integrando-o a diferentes camadas de tecnologias, como imagens de satélite e descargas atmosféricas.
\end{abstract}

\section{Introdução}

O radar meteorológico é uma ferramenta capaz de coletar dados que permitem a identificação de nuvens carregadas e estimar a intensidade da precipitação associada às mesmas, assim como determinar a estrutura vertical de tempestades (e seu deslocamento) e seu potencial de causar condições de tempo severas [Moreno, Mujika e Segura, 2014].

Outro tipo de visualização de fenômenos meteorológicos são as imagens de satélites, que fornecem uma visão global e consistente da organização das características atmosféricas em uma numerosa variedade de escalas sobre grandes áreas [Georgiev, Santurette e Maynard, 2016]. Também é relevante realizar o monitoramento da ocorrência de descargas atmosféricas provocadas pelas tempestades, para entender a formação e os impactos de nuvens e tempestades [Radunovic, Vujicic e Balota, 2017].

Nesse âmbito, o Centro Gestor e Operacional do Sistema de Proteção da Amazônia (Censipam) desenvolveu o sistema SipamHidro (Sistema Integrado de Monitoramento e Alerta Hidrometeorológico), que pode ser definido como um conjunto de tecnologias, metodologias e procedimentos operacionais, integrados em uma plataforma de banco de dados que fornece espacialização, análise e previsão das condições hidrológicas e meteorológicas na Amazônia [Censipam, 2019].

Em vista disso, o módulo do Radar Meteorológico no SipamHidro tem como objetivo monitorar as chuvas e tempestades identificadas pelos radares, integrando dados meteorológicos de diferentes fontes, além de adicionar informações de apoio, como imagens de satélite e ocorrências de descargas atmosféricas.

\section{Dados e Metodologia}

O módulo do Radar Meteorológico foi desenvolvido como um modelo cliente-servidor por meio de um serviço web, cujo principal componente do cliente (navegador) é o mapa do Google Maps. Por meio da API do Google Maps, o mapa é criado e controlado, onde são exibidas todas as camadas e imagens com informações, além de se comunicar com 
scripts em JavaScript, que são responsáveis pela aquisição dos dados nos servidores e sistemas de arquivos.

Já o processamento dos dados dos radares ocorre como mostra a Figura 1. Foram utilizadas quatro ferramentas do projeto chamado LROSE ${ }^{1}$ : Radx2Grid (transforma coordenadas de uma grade esférica na qual os dados do radar terrestre são coletados em uma grade regular), PrecipAccum (calcula a taxa de precipitação), Mdv2Vil (estima o conteúdo líquido de água em cada coluna vertical) e PrintMdv (transforma os dados de uma grade regular para texto).

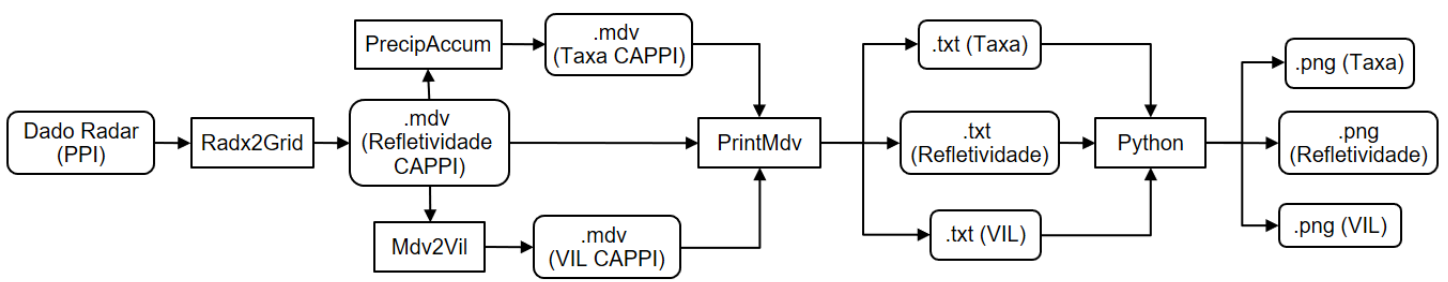

Figura 1. Esquema de processamento dos dados do radar em figuras.

O Radx2Grid gera dados - a partir dos dados do radar meteorológico em PPI (indicador de posição de plano) - de refletividade em CAPPI (indicador de posição do plano de altitude), que, em seguida, são processados pelo PrecipAccum e Mdv2Vil e geram, respectivamente, dados de taxa de precipitação e VIL. Esses dados são convertidos de .mdv (volume de dados meteorológicos) para texto. Depois, os dados em texto são processados por um script em python - utilizando as bibliotecas Numpy, Matplolib e Basemap - responsável por transformá-los em imagens, que são armazenadas em um sistema de arquivos disponibilizado para o SipamHidro por meio de uma API e possibilitar ser acessado como uma camada de visualização no sistema. Todo esse processo é realizado automaticamente a cada 12 minutos, que é o intervalo de tempo que os radares estão configurados para realizar as varreduras de $240 \mathrm{~km}$.

\section{Radar Meteorológico}

Este módulo disponibiliza o monitoramento de tempestades em uma área de cobertura com raio de $240 \mathrm{~km}$ a partir dos radares meteorológicos instalados nos municípios de Belém, Boa Vista, Cruzeiro do Sul, Macapá, Manaus, Porto Velho, Santarém, São Gabriel de Cachoeira, São Luís, Tabatinga e Tefé, os quais são representados por ícones posicionados sobre estas cidades no mapa exibido na página inicial do produto. A interface também oferece um menu flutuante à esquerda, contendo uma lista de seleção com todos os radares. Se o radar estiver inativo por mais de três dias consecutivos, o seu ícone não aparecerá no mapa, mas, ainda assim, estará na lista - como é o caso de Belém e São Luís.

Ao clicar em um dos ícones no mapa ou selecionar um radar na lista, as suas últimas cinco varreduras realizadas são inseridas em uma lista no menu (em horário local do radar selecionado) e a mais recente é apresentada espacializada no mapa. É possível selecionar cada uma delas individualmente por meio da lista ou animá-las para melhor compreender o seu comportamento ao longo do tempo, além de poder ajustar a sua opacidade. Quando o radar está atrasado (mais de três horas), um aviso de "Atrasado" é 
exibido na lista de varreduras, mas ainda é possível visualizar as últimas cinco realizadas - assim como os radares inoperantes. Atualmente, apenas a variável de refletividade máxima está disponível para visualização. A Figura 2 mostra um exemplo da imagem da varredura de um dos radares, com o menu flutuante aumentado.

Também está disponível a opção de previsão de tempestades, na qual é possível escolher um ou mais horizontes de tempo de previsão. Os polígonos de tempestades referentes ao horizonte selecionado são exibidos no mapa, podendo-se obter mais informações acerca de cada tempestade, como altura do topo da nuvem, massa de granizo e área projetada, ao clicar sobre eles. Por fim, há a funcionalidade de corte transversal, cujo objetivo é obter o perfil vertical das tempestades que estiverem presentes na linha de corte desenhada pelo usuário. Todas as informações do módulo são atualizadas a cada 12 minutos, para acompanhar o processamento dos novos dados coletados dos radares.

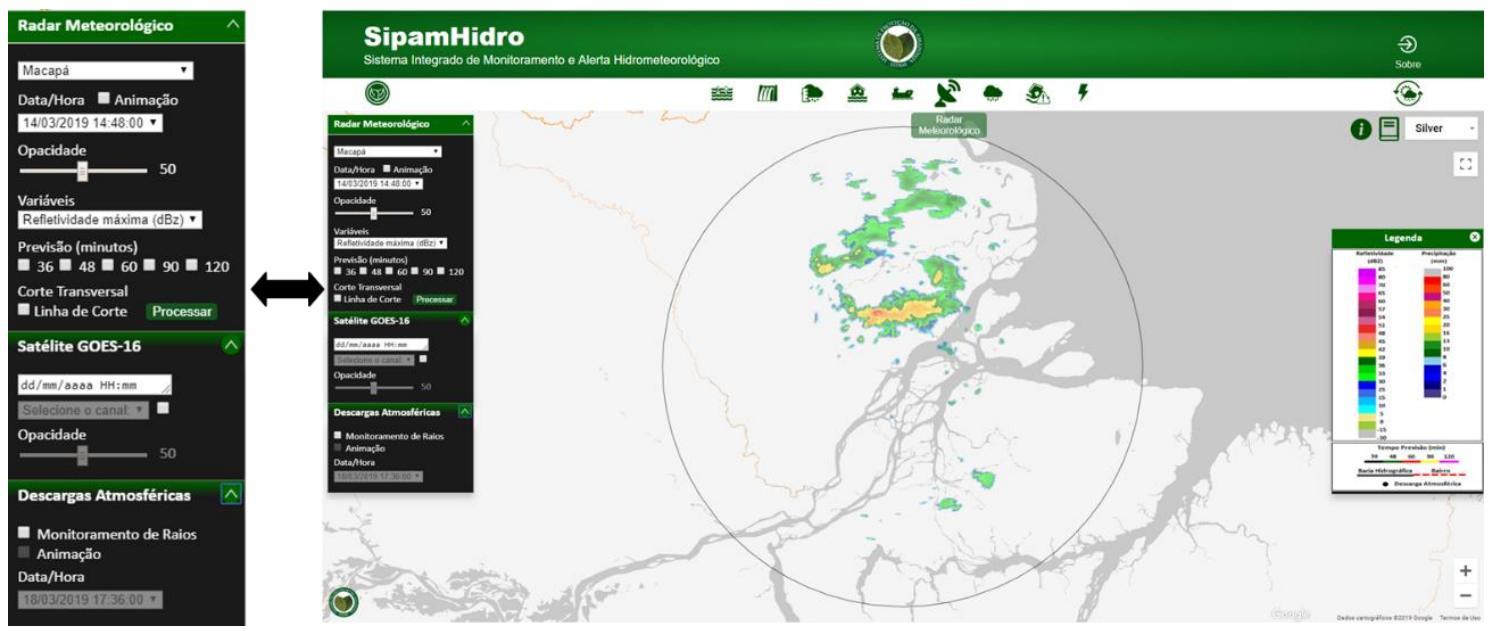

Figura 2. Varredura do radar de Macapá do dia 14/03/2019 às 14 h48 (hora local).

\subsection{Camadas Auxiliares}

Ao habilitar a função do Satélite GOES-16, pode-se escolher um dos canais disponíveis (infravermelho, vapor d'água e visível). Em seguida, a imagem do canal correspondente com o horário mais próximo ao do radar escolhido é apresentada. Entretanto, se o radar selecionado estiver atrasado ou inoperante, a imagem de satélite torna-se indisponível, visto que apenas as mais recentes são armazenadas.

Quando a funcionalidade de Descargas Atmosféricas é habilitada, o mapa exibe as ocorrências de raios em vermelho. Em seguida, é disponibilizada uma lista de horários sincronizados aos do radar selecionado e de mais 1 hora na frente. De maneira semelhante às imagens do radar, é possível visualizar cada um dos horários das descargas atmosféricas individualmente ou animá-los. Se a animação for selecionada, as varreduras do radar automaticamente acompanham as ocorrências de descargas - de acordo com os horários - até alcançar a imagem da última varredura, que permanece fixa enquanto são exibidas as ocorrências de descargas da hora subsequente. A Figura 3 mostra um exemplo no qual são utilizadas as duas camadas auxiliares juntamente com a camada do radar.

\section{Conclusões}

O novo módulo do Radar Meteorológico contido no SipamHidro é uma ferramenta relevante na identificação e monitoramento de tempestades, integrando essas informações 
com dados auxiliares, como imagens de satélite e ocorrências de descargas atmosféricas. Isso potencializa a análise e a agregação de conhecimento por parte dos especialistas que podem melhorar o estudo dos dados oferecidos pelos radares e suas consequências, aprimorando as tomadas de decisões. Além disso, os dados dos radares meteorológicos poderão ser empregados em outros módulos do SipamHidro, por exemplo, na identificação da taxa média de chuva nos bairros dos municípios nos raios de coberturas dos radares e na previsão de chuva e tempestades em áreas de navegação fluvial.

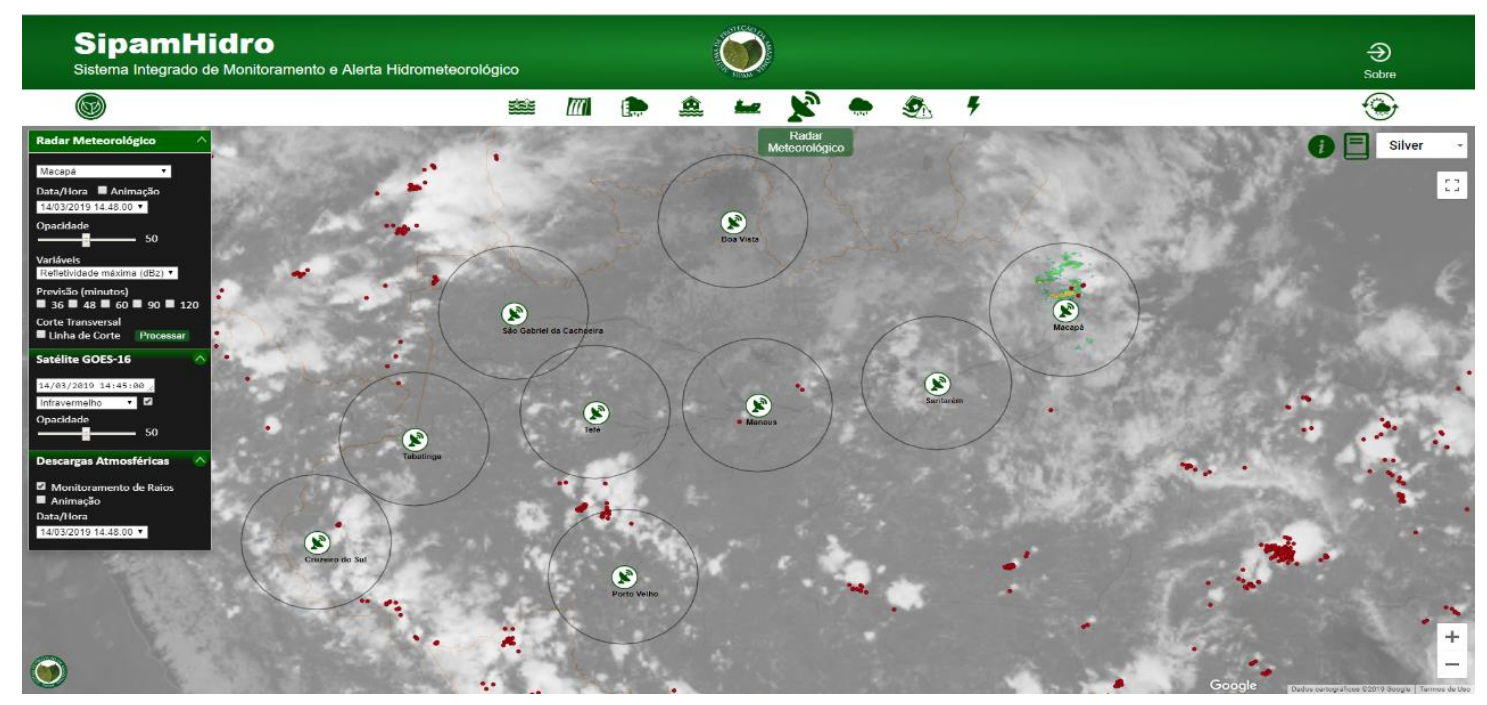

Figura 3. Camadas do Satélite GOES-16 e de Descargas Atmosféricas (em vermelho) em conjunto com a camada do radar.

\section{Agradecimentos}

O presente trabalho foi realizado com o apoio do Censipam e o $\mathrm{CNPq}-\mathrm{Brasil}$. Os autores também gostariam de agradecer ao SOS Amazônia, ao Laboratório STORM-T da USP, ao INPE e a NOAA pelo fornecimento dos dados.

\section{Referências}

Censipam. (2019) Apresentação do Censipam. Disponível em: $<$ http://www.sipam.gov.br/noticias/apresentacao-do-censipam $>$. Acesso em: $12 \mathrm{de}$ jan. de 2019.

Georgiev, C. Santurette, P. e Maynard, K. (2016), Weather Analysis and Forecasting, Elsevier Inc, $2^{\mathrm{a}}$ edição.

Moreno, A. Mujika, A. e Segura, A. Visual analytics of multi-sensor weather information georeferenciation of Doppler weather radar and weather stations. In: 2014 International Conference on Information Visualization Theory and Applications (IVAPP). 2014, Lisboa, Portugal: IEEE, p. 329-336.

Radunovic, P. Vujicic, T. e Balota, A. (2017). Web application for lightning activity monitoring system (LAMS). In: $201712^{\text {th }}$ Iberian Conference on Information Systems and Technologies (CISTI). 2017, Lisboa, Portugal: IEEE, p. 1-4. 